Series A

I. MATHEMATICA

$336 / 6$

ÜBER DIE VERWENDUNG VON

DIFFERENTIALINVARIANTEN BEI GEWISSEN

FUNKTIONENFAMILIEN UND

DIE ÜBERTRAGUNG EINER DARAUF

GEGRÜNDETEN METHODE AUF PARTIELLE DIFFERENTIALGLEICHUNGEN VOM ELLIPTISCHEN TYPUS

vON

ERNST PESCHL

H E L S I N K I 1963

S U O M A L A I N E N T I D E A K A T E M I A 
Am 11. Januar 1963 vorgelegt von P. J. Myrberg und Olli Lehto 


\section{Über die Verwendung von Differentialinvarianten bei gewissen Funktionen- familien und die Übertragung einer darauf gegründeten Methode auf partielle Differentialgleichungen vom elliptischen Typus}

1. Die geometrische Funktionentheorie hat mit grossem Erfolg differentialgeometrische Grundbegriffe methodisch verwendet. Es sei in diesem Zusammenhange nur an die Nevanlinna-Ahlforssche Theorie der Werteverteilung erinnert. Die Schule Nevanlinna-Ahlfors hat diese Gedanken systematisch weiter ausgebaut. Auch von anderen wurden wiederholt und mit grossem Erfolg differentialgeometrische Betrachtungen in der Funktionentheorie verwendet. Ich möchte heute einen kurzen Überblick über eine spezielle Methode geben, die in der Funktionentheorie das Hauptaugenmerk auf die Gewinnung von scharfen Ungleichungen für gewisse Differentialinvarianten richtet, und von der man andererseits zeigen kann, dass sich ihr Kern - lösgelöst von den ursprünglichen Differentialinvarianten - auf eine grosse Klasse partieller Differentialgleichungen vom elliptischen Typus übertragen lässt. Diese letzteren Ergebnisse habe ich erst in jüngster Zeit gewonnen. Wegen der Tragweite erscheint es mir nützlich, die zugrundeliegenden Gedanken einmal im Zusammenhang darzustellen.

\section{Holomorphe Abbildungen der hyperbolischen Ebene in die euklidische Ebene}

2. Als einfachste Differentialinvarianten bieten sich im Falle der holomorphen Abbildung eines beschränkten Gebietes in die euklidische Ebene an:

$$
\alpha=\lg \frac{|d w|}{d s}, \quad \gamma=\delta_{1} \alpha,
$$

worin $d s$ das Linienelement der hyperbolischen Metrik dieses Gebietes und $\delta_{1}$ den ersten Beltrami-Operator in dieser Geometrie bedeuten. Um diese Methode an einem besonders einfachen Beispiel zu zeigen, werde die Familie der unverzweigten holomorphen Abbildungen der offenen Kreisscheibe $z \bar{z}<1$ betrachtet. Für sie gilt der folgende 
Satz 1. Sei $w(z)$ holomorph und $w^{\prime}(z) \neq 0$ in $z \bar{z}<1, \alpha=\lg \left\{\left|w^{\prime}\right|(1-z \bar{z})\right\}$, seien ferner $\alpha_{0}=\sup _{z \bar{z}<1} \alpha, \alpha_{1}=\alpha(0)$, dann gelten die folgenden Ungleichungen $(2.2 . \mathrm{a}, \mathrm{b}) \quad-\frac{2 a r}{1-r}+\lg \frac{1+r}{1-r} \leqq \alpha-\alpha_{1} \leqq \frac{2 a r}{1+r}+\lg \frac{1-r}{1+r}$, oder

$$
\frac{1}{(1-r)^{2}} e^{-\frac{2 a r}{1-r}} \leqq \frac{\left|w^{\prime}(z)\right|}{\left|w^{\prime}(0)\right|} \leqq \frac{1}{(1+r)^{2}} e^{\frac{2 a r}{1+r}},
$$

worin $|z|=r$ und $a \geqq 1$ durch

$$
1-a+\lg a=\alpha_{1}-\alpha_{0}
$$

eindeutig bestimmt ist, ferner

$$
|w(z)-w(0)| \leqq \frac{1}{2 a} e^{\alpha_{1}}\left(e^{\frac{2 a r}{1+r}}-1\right),
$$

sowie für den grössten Radius $\varrho(r)$ einer Kreisscheibe $|w-w(0)|<\varrho$, die vom $w$-Bild der Kreisscheibe $|z|<r$ schlicht überdeckt wird:

$$
\varrho(r) \geqq \frac{1}{2 a} e^{\alpha_{1}}\left(1-e^{-\frac{2 a r}{1-r}}\right) .
$$

Die Abschätzungen nach oben in (2.2.b), (2.3.b), (2.5) gelten nur für

$$
0 \leqq r \leqq r^{*}=\frac{a-1}{a+1} .
$$

Die obigen Ungleichungen sind scharf, wie man an der Schrankenfunktion

$$
w=\frac{1}{2} e^{1+\alpha_{0}} e^{a \frac{z-1}{z+1}}
$$

erkennt, [6], [7].

Aus der zuletzt genannten Ungleichung (2.6) ergibt sich für $r \rightarrow 1$ :

$$
\varrho(1) \geqq \frac{1}{2 a} e^{\alpha_{1}},
$$

und da man eine Punktfolge $z_{n},\left|z_{n}\right|<1$, mit $\alpha\left(z_{n}\right) \rightarrow x_{0}$ wählen und die Betrachtung auf

$$
\omega(\zeta)=w\left(\frac{\zeta+z_{n}}{1+\bar{z}_{n} \zeta}\right)
$$

anwenden darf, hat man dann in (2.9): $\alpha_{1} \rightarrow \alpha_{0}$ und $a \rightarrow 1$, daher 


$$
\varrho(1) \geqq \frac{1}{2} e^{\alpha_{0}}-\varepsilon,
$$

und somit den

Satz 2. (Bloch-Landau-Ahlfors). Sei $w=z+\ldots$ holomorph und $w^{\prime} \neq 0$ im Einheitskreis $z \bar{z}<1$, dann überdeckt das dadurch erzeugte Bild des Einheitskreises mindestens eine Kreisscheibe von einem Radius, der beliebig wenig unter $1 / 2$ liegt, $\left.[1]^{1}\right)$.

3. Zum Beweise von Satz 1 verwenden wir:

und bilden:

$$
d s^{2}=\frac{d z d \bar{z}}{(1-z \bar{z})^{2}}
$$

$$
\alpha=\lg \left\{\left|w^{\prime}\right|(1-z \bar{z})\right\}, \quad \beta=(1-z \bar{z}) \alpha_{z}=\frac{1}{2} \frac{w^{\prime \prime}}{w^{\prime}}(1-z \bar{z})-\bar{z},
$$

$\gamma=\beta \bar{\beta}$. Wir betrachten nun die Funktion $v=\gamma-f(\alpha)$ für eine zweimal stetig differenzierbare, jedoch zunächst sonst beliebige Funktion $f$ und berechnen den zweiten Beltrami-Operator $\delta_{2} v=(1-z \bar{z})^{2} v_{z \bar{z}}$. Man erhält:

$$
\delta_{2} v=\frac{1}{\gamma} \delta_{1} v+\frac{1}{\gamma}\left(1+f^{\prime}\right)\left(\delta_{1}(v, \alpha)+\delta_{1}(\alpha, v)\right)+D_{0}+D_{1} v
$$

mit

$$
\begin{gathered}
\delta_{1}(v, \alpha)=(1-z \bar{z})^{2} v_{z} \alpha_{\bar{z}}=\overline{\delta_{1}(\alpha, v)}, \\
D_{0}=-f\left(2+f^{\prime \prime}\right)+\left(f^{\prime}+1\right)\left(f^{\prime}+2\right), \\
D_{1}=-\left(2+f^{\prime \prime}\right) .
\end{gathered}
$$

Übrigens kann man für die weiteren Betrachtungen zunächst die Voraussetzungen über $w(z)$ als im abgeschlossenen Einheitskreis erfüllt ansehen und die obigen Ungleichungen unter diesen einschränkenden Bedingungen beweisen. Betrachtet man nachträglich die Funktion

$$
w_{1}(z)=\frac{1}{\varrho} w(\varrho z) \quad \text { mit } \quad 0<\varrho<1, \quad \varrho \rightarrow 1,
$$

so erhält man dann hieraus den ursprünglichen Satz 1 .

1) Es sei hier ausdrücklich darauf hingewiesen, dass unsere Methode zwar scharfe Abschätzungen für die betreffende Funktionenfamilie (im Sinn von Satz 1) und andere ähnliche Familien liefert, dass jedoch die dadurch erzielte Abschätzung der verschiedenen Bloch-Konstanten (im Sinne von Satz 2) nicht scharf ist, sondern sich nur als gleichwertig mit der derzeit besten von Ahlfors (Methode der Stützmetrik) angegebenen erweist, [1], [5], [6]. 
Man sucht nun $D_{0}=0$ so zu lösen, dass die folgende Anfangsbedingung erfüllt wird: Für $\alpha \rightarrow-\infty$ solle $f \rightarrow \infty$ gehen, jedoch möglichst schwach, d. h. $f=f(\alpha)$ solle die untere Grenze aller Integralkurven durch einen festen Punkt mit dieser Eigenschaft sein. Der Ansatz $f^{\prime}=(f-1) y(f)$ führt übrigens die Differentialgleichung $D_{0}=0$ in eine separierbare Form über, so dass man sämtliche Lösungen von $D_{0}=0$ elementar gewinnen kann. Die eben genannte Kennzeichnung sondert aus allen Lösungen die folgende einparametrige Kurvenschar aus:

$$
\alpha-\lambda=-\sqrt{f}+\lg (1+\sqrt{f}) \quad \text { für } \alpha \leqq \alpha_{0}<\lambda_{0} \leqq \lambda .
$$

4. Was man jetzt zu tun hat, ist im wesentlichen, dass man die bei Annäherung an den Rand bestehende Ungleichung $v<0$ auch auf das Innere auszudehnen hat. Das dabei angewandte Beweisprinzip lässt sich zusammenfassen im folgenden

Satz 3. Sei $w(z)$ holomorph und $w^{\prime} \neq 0$ in $z \bar{z} \leqq 1$. Sei $f(\alpha, \lambda)$ für $\lambda_{0} \leqq \lambda \leqq \lambda_{1}$ eine einparametrige Schar von Lösungen von $D_{0}=0$, welche folgende Bedingungen erfüllt:

1) $f(\alpha, \lambda)$ sei definiert für $\alpha \leqq \alpha_{0}$,

2) $D_{1} \neq 0$

3) $f_{\lambda}>0$ und

4) $m=\frac{f i n}{\alpha \leqq \alpha_{0}} f\left(\alpha, \lambda_{0}\right)>0$.

Setzt man $v(z, \lambda)=\gamma-f(\alpha, \lambda)$, so sei bei Annäherung an den Rand des Einheitskreises

$$
\begin{gathered}
v\left(z, \lambda_{0}\right)<0 \quad \text { (»Randbedingung»), } \\
v\left(z, \lambda_{1}\right) \leqq 0 \text { für } z \bar{z} \leqq 1 \quad \text { (»Feldbedingung»), }
\end{gathered}
$$

dann gilt $v\left(z, \lambda_{0}\right) \leqq 0$ für alle $z \bar{z}<1$.

Der Beweis dieses Satzes stützt sich auf eine Modifikation des Maximumprinzips. Wäre nämlich an einer Stelle $z_{0}$ im Innern des Einheitskreises $v\left(z_{0}, \lambda_{0}\right)>0$, so könnte man ein $\lambda^{*}, \lambda_{0}<\lambda^{*}<\lambda_{1}$ und ein $z^{*},\left|z^{*}\right|<1$, finden, so dass $v\left(z^{*}, \lambda^{*}\right)=0$ und $v\left(z, \lambda^{*}\right) \leqq 0$ für $z \bar{z} \leqq 1$. Für ein geeignetes genügend kleines $\varepsilon(\neq 0)$ würde dann eine Stelle $z^{* *}$ im Innern des Einheitskreises zu finden sein, worin $v\left(z^{* *}, \lambda^{*}+\varepsilon\right)$ ein Maximum hätte und gleichzeitig $\delta_{2} v>0$ wäre, womit man dann den Widerspruch hat, [3].

5. Aus diesem Satz 3 ergibt sich für die Funktionenfamilie des Satzes 1 die »scharfe» Abschätzung (5.2), nämlich zunächst

(5.1) $\gamma=\beta \bar{\beta} \leqq f\left(\alpha, \lambda_{0}\right) \quad$ mit $f\left(\alpha, \lambda_{0}\right)$ aus (3.5) und jedes $\lambda_{0}>\alpha_{0}$, 
daher auch:

$$
\gamma \leqq f\left(\alpha, \alpha_{0}\right)
$$

und für die obige Schrankenfunktion (2.8) gilt hierin das Gleichheitszeichen, [6], [7].

Aus (5.2) gewinnt man dann wegen $\gamma=\delta_{1} \alpha$ durch Integration der Ungleichung

$$
\frac{1}{\sqrt{f\left(\alpha, \lambda_{0}\right)}}\left|\frac{\partial \alpha}{\partial r}\right| \leqq \frac{2}{1-r^{2}},
$$

die Ungleichungen (2.2), (2.3) für $\left|w^{\prime}(z)\right|$ und durch einen nochmaligen Integrationsschritt die Ungleichungen (2.5), (2.6) für $w(z)$.

6. Es seien noch zwei weitere wichtige Beispiele von Funktionenfamilien genannt, die in derselben Weise behandelt werden können:

1) Die Familie der in $z \bar{z} \leqq 1$ holomorphen Funktionen, deren Ableitung dort nicht verschwindet und die den Einheitskreis auf ein Gebiet abbilden, dessen Rand eine euklidische Krümmung $k \geqq k_{0}$ hat (für $k_{0}<0$ braucht das Bildgebiet nicht schlicht zu sein), [4]. In diesem Fall gewinnt man aus der Beziehung

$$
\lim _{z \rightarrow z_{0}}(1-\beta \bar{\beta}) e^{-\alpha}=k, \quad|z|<1, \quad\left|z_{0}\right|=1,
$$

die Anfangsbedingung für die auszuwählende "ntegralkurve von $D_{0}=0$ :

$$
\lim _{\alpha \rightarrow-\infty} f(\alpha)=1,
$$

$$
\lim _{\alpha \rightarrow-\infty} e^{-\alpha}(f-1) \geqq-k_{0} \text {. }
$$

2) Die Familie der in $z \bar{z}<1$ holomorphen Funktionen, für die die Vielfachheit $v$ der Nullstellen ihrer Ableitung - soweit solche überhaupt auftreten - der Forderung $v \geqq v_{0}$ unterworfen wird, und $v_{0}$ eine für alle Funktionen der Familie gleiche feste Zahl ist. Ebenso wie in dem Hauptbeispiel ist es keine Beschränkung der Allgemeinheit, wenn man zunächst die Ungleichung für den Fall herleitet, dass man die Holomorphie im abgeschlossenen Einheitskreis und das Nichtverschwinden der Ableitung auf den Rand des Einheitskreises fordert. Sodann hat man in diesem Fall die nur endlich vielen Nullstellen der Ableitung noch in die Randbetrachtung mit einzubeziehen. Man zeigt leicht, dass für die Annäherung an eine Nullstelle von $w^{\prime}(z)$ von der Vielfachheit $v$ gilt:

$$
\beta \bar{\beta}=e^{-\frac{2}{v} \alpha} e^{o(1)} \text {. }
$$


Um der Randbedingung des Satzes 3 zu genügen, hat man hier Integralkurven zu wählen, die für $\alpha \rightarrow-\infty$ der Bedingung genügen

$$
f=e^{-b \alpha} e^{O_{(1)}} \quad \text { mit } \frac{2}{v} \leqq \frac{2}{v_{0}}<b .
$$

Ausserdem fügt man wieder die Bedingung $\alpha \leqq \alpha_{0}$ hinzu. Die bestmögliche Abschätzung liefert die Integralkurve mit $b=2 / v_{0}, \quad[6],[10]$.

7. Grundsätzlich lässt sich zeigen, dass man jede Integralkurve von $D_{0}=0$ in ein geeignetes Feld von Integralkurven einbetten kann (im Sinne des Satzes 3). Hier taucht die Frage auf, ob so zu jeder Integralkurve von $D_{0}=0$ eine Familie von Funktionen $w(z)$ (entsprechend der "Randbedingung» von Satz 3) gehört. Man sieht aber bereits an dem Beispiel einer Integralkurve mit $f=e^{-b \alpha} e^{O(1)}$ (für $\alpha \rightarrow-\infty$ ), worin $2 / b$ keine natürliche Zahl ist, dass die Holomorphiebedingung für $w(z)$ nicht mehr erfüllt werden kann. Diese Überlegung gab den ersten Anlass, den Satz 3 und den anschliessenden ersten Integrationsschritt (des Satzes 1) ohne Bezug auf holomorphe Funktionen lediglich für Lösungen der partiellen Differentialgleichung $\delta_{2} \alpha=C \quad(C$ eine negative Konstante $)$ zu übertragen, wobei man isolierte Singularitäten eines bestimmten Verhaltens noch zulässt. Es zeigt sich, dass ein solches Programm voll durchführbar ist und dann grundsätzlich in der Tat zu jeder Integralkurve von $D_{0}=0$ eine entsprechende Familie von Lösungen $\alpha$ der partiellen Differentialgleichung $\delta_{2} \alpha=C$ zu finden ist, für die zugehörige sscharfe» Abschätzungen im Sinne der ersten Ungleichungen (2.2) des Satzes 1 hergeleitet werden können, [10].

\section{Verallgemeinerung auf nichteuklidische Metrik in der Bildebene}

8. In Verallgemeinerung ([2], [6], [7]) des ersten Ansatzes in I werde in der $z$-Ebene und in der $w$-Ebene (Bildebene) zunächst je eine beliebige Metrik zugrundegelegt:

$$
d s_{1}^{2}=e^{2 a} d z d \bar{z}, \quad d s_{2}^{2}=e^{2 b} d w d \bar{w},
$$

und die zugehörige Differentialinvariante

$$
\alpha=\lg \frac{d s_{2}}{d s_{1}}=b-a+\lg \left|w^{\prime}\right|
$$

betrachtet. Wir bedienen uns wieder des ersten und zweiten BeltramiOperators für die Metrik in der $z$-Ebene: 


$$
\delta_{1} u=e^{-2 a} u_{z} u_{\bar{z}}, \quad \delta_{2} u=e^{-2 a} u_{z \bar{z}} .
$$

Man erhält sofort

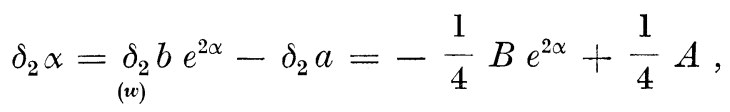

worin $\delta_{2} b$ den zweiten Beltrami-Operator in bezug auf die $w$-Ebene, (w)

sowie $A$ bzw. $B$ die Gausssche Krümmung zum Linienelement $d s_{1}$ bzw. $d s_{2}$ bedeuten.

Um die in I entwickelte Methode auf diesen Fall übertragen zu können, hat man die folgenden drei Teilaufgaben $\mathrm{zu}$ erfüllen:

(1) Berechnung von $\delta_{2} v$ für $v=\gamma-f(\alpha), \gamma=\delta_{1} \alpha$. Es ergibt sich:

(8.5) $\delta_{2} v=$

$$
\frac{1}{\gamma} \delta_{1} v+\frac{1}{\gamma}\left(f^{\prime}-\frac{1}{4} A+\frac{1}{4} B e^{2 \alpha}\right)\left(\delta_{1}(v, \alpha)+\delta_{1}(\alpha, v)\right)+D_{0}+D_{1} v
$$

mit

$$
\delta_{1}(v, \alpha)=e^{-2 \alpha} v_{z} v_{\bar{z}}=\overline{\delta_{1}(\alpha, v)}
$$

$$
-f\left(f^{\prime \prime}-\frac{A}{2}+B e^{2 \alpha}\right)+\left(f^{\prime}-\frac{1}{4} A+\frac{1}{4} B e^{2 \alpha}\right)\left(f^{\prime}-\frac{1}{2} A+\frac{B}{2} e^{2 \alpha}\right),
$$

$$
D_{1}=-\left(f^{\prime \prime}-\frac{A}{2}+B e^{2 \alpha}\right) \text {. }
$$

Die Durchführung des Beweisprinzips von Satz 3 verlangt, dass $f(\alpha)$ der Differentialgleichung $D_{0}=0$ genügt und, dass die Gaussschen Krümmungen $A$ und $B$ konstant sein müssen.

(2) Die Untersuchung des Randverhaltens von $\alpha$. Dabei kann es sich wiederum entweder um Randpunkte des Grundgebietes der Metrik handeln oder um im Innern dieses Gebietes gelegene isolierte Punkte. Legt man der Einfachheit halber wieder den Einheitskreis der z-Ebene zugrunde, so verbleibt nur der Fall, dass $A<0$ ist, wobei wir die Normierung $A=-4$ wählen können. Natürlich kann man diese Metrik auch für den Fall allgemeinerer Grundgebiete behandeln. In der Bildebene haben wir nunmehr die drei Hauptfälle (in normierter Form): $B=4 \varepsilon$, $\varepsilon=-1,0,+1$, wobei der Fall $\varepsilon=0$ in I behandelt wurde, der Fall $\varepsilon=-1$ (in der Normierung $w \bar{w}<1$ ), die im Einheitskreis holomorphen und beschränkten Funktionen, der Fall $\varepsilon=+1$ die im Einheitskreis meromorphen Funktionen betrifft, wobei jetzt die gesamte Riemannsche 
Zahlenkugel Grundgebiet der Metrik $d s_{2}$ wird. Das Randverhalten in isolierten inneren Punkten der Metrik wird wiederum in derselben Weise studiert wie in I, [2], [6], [7].

(3) Die Integration der Differentialgleichung $D_{0}=0$ lässt sich vollständig durchführen, [2], [8]. Die Tatsashe, dass auch hier zwischen Randverhalten für eine bestimmte Funktionenfamilie und Anfangsbedingungen für eine Integralkurve eine enge Beziehung besteht und dass auch hier wiederum für analog gebildete Funktionenfamilien scharfe Abschätzungen zu erwarten waren, gab wertvolle Hinweise für die Auffindung von Lösungen dieser Differentialgleichung, indem man die vermuteten Schrankenfunktionen aus speziellen konformen Abbildungen berechnet und durch Übergang zu den Invarianten $\alpha$ und $\gamma$ schliesslich einparametrige Scharen von Integralen findet. Von diesen ausgehend gelangt man durch analytische Fortsetzung in der Ebene des Scharparameters über das Komplexe hinweg zu weiteren einparametrigen Scharen von reellen Integralen. Einige partikuläre Zwischenintegrale sind von der Form $(\sqrt{f}=g)$ :

$$
g^{\prime}=\sum_{\nu=-1}^{\infty} a_{\nu}(\alpha) g^{\nu}
$$

Dieser allgemeine Ansatz liefert dann das allgemeine Zwischenintegral, das man nachträglich auch mit der Methode des integrierenden Faktors erhält, wodurch man schliesslich einen Überblick über sämtliche Lösungen erhält, [2], [8].

Eine genaue Überprüfung zeigt die vollständige Übertragbarkeit des Satzes 3. Daraus gewinnt man dann eine grosse Fülle von wertvollen Aussagen für analog gebildete Funktionenfamilien wie in I. Im einzelnen ist hierzu zu bemerken, dass hier eine viel grössere Zahl von interessanten Funktionenfamilien auftritt, insbesondere für $\varepsilon=-1$. Ebenso ist der Fall $\varepsilon=+1$ besonders bemerkenswert, da die im Einheitskreis meromorphen Funktionen damit erfasst werden. Die hier erzielten Ergebnisse und Abschätzungen (analog zu (5.2), (2.2), (2.5)):

$$
\begin{aligned}
& \gamma-f(\alpha) \leqq 0, \quad h_{1}\left(r, \alpha_{1}\right) \leqq \alpha \leqq h_{2}\left(r, \alpha_{1}\right), \\
& \left|\frac{w(z)-w(0)}{1+\varepsilon w(0) w(z)}\right| \leqq h_{3}\left(r, \alpha_{1}\right),
\end{aligned}
$$

sowie für einen analog zu (2.6) definierten »Radius» $\varrho(r)$ einer Kreisscheibe

$$
\mid \frac{w-w(0)}{1+\varepsilon w(0) w}<\varrho
$$

sind in den weiteren funktionentheoretischen Folgerungen noch keineswegs voll ausgewertet, [2], [6], [7]. 


\section{III. Übergang zu partiellen Differentialgleichungen}

9. Prüft man - im Hinblick auf die Schlussbemerkung in I.7 die Frage nach der Übertragbarkeit dieses Verfahrens auf die reelle Analysis, so ist die Erkenntnis von entscheidender Wichtigkeit, dass von der speziellen Natur der Invarianten $\alpha$ in den Kalkül von $\delta_{2} v$ eigentlich nichts ausser der Differentialgleichung $\delta_{2} \alpha=L(\alpha)$ (mit speziellem $L(\alpha)$ ) eingeht. Dies gibt Veranlassung, den Ansatz in dieser Richtung zu verallgemeinern.

In einem Grundgebiet der $z$-Ebene sei die Metrik

$$
d s^{2}=e^{2 a} d z d \bar{z}
$$

mit konstanter Gaussscher Krümmung $A<0$ gegeben. Unter Verwendung des ersten und zweiten Beltrami-Operators dieser Metrik:

$$
\gamma=\delta_{1} u=e^{-2 a} u_{z} u_{\bar{z}}, \quad \delta_{2} u=e^{-2 a} u_{z \bar{z}}
$$

werde nunmehr die Differentialgleichung vom elliptischen Typus

$$
\delta_{2} u=L(u, \gamma)
$$

zugrundegelegt. Hierbei soll die Funktion $L$ für beliebige $u, \gamma$ reell-analytisch sein. Die in II formulierten drei Teilaufgaben, deren Bewältigung zur Übertragung der Methode erforderlich ist, führen im einzelnen zu folgenden Betrachtungen:

1. Die Berechnung von $\delta_{2} v$ für $v=\gamma-f(u)$ liefert folgendes Ergebnis:

$$
\delta_{2} v=\frac{1}{\gamma} \delta_{1} v+\left(L_{\gamma}+\frac{1}{\gamma}\left(f^{\prime}-L\right)\right)\left(\delta_{1}(u, v)+\delta_{1}(v, u)\right)+D
$$

mit

$$
\delta_{1}(v, u)=e^{-2 a} v_{z} u_{\bar{z}}=\overline{\delta_{1}(u, v)},
$$

und

$$
\begin{gathered}
D= \\
{\left[-(f+v)\left(f^{\prime \prime}-\frac{A}{2}-2\left(L_{u}+L_{\gamma} f^{\prime}\right)\right)+\left(f^{\prime}-L\right)\left(f^{\prime}-2 L\right)\right]_{\gamma=f+v}} \\
=D_{0}+D_{1} v+\ldots, \\
\text { 4) } \quad D_{0}=-f\left(f^{\prime \prime}-\frac{A}{2}-2\left(L_{u}^{0}+f^{\prime} L_{\gamma}^{0}\right)\right)+\left(f^{\prime}-L^{0}\right)\left(f^{\prime}-2 L^{0}\right), \\
\text { 5) } D_{1}=-f^{\prime \prime}+\frac{A}{2}+2 L_{u}^{0}-L_{\gamma}^{0}\left(f^{\prime}-4 L^{0}\right)+2 f\left(L_{u \gamma}^{0}+f^{\prime} L_{\gamma \gamma}^{0}\right),
\end{gathered}
$$

wobei der obere Index 0 bedeutet: $[\ldots]_{\gamma=f}$. 
Der wichtige Sonderfall

$$
L_{\gamma}=0, \quad L=L(u),
$$

liefert:

$$
D=-(f+v)\left(f^{\prime \prime}-\frac{1}{2} A-2 L^{\prime}\right)+\left(f^{\prime}-L\right)\left(f^{\prime}-2 L\right)=D_{0}+D_{1} v
$$

mit

$$
\begin{gathered}
D_{0}=-f\left(f^{\prime \prime}-\frac{1}{2} A-2 L^{\prime}\right)+\left(f^{\prime}-L\right)\left(f^{\prime}-2 L\right), \\
D_{1}=-f^{\prime \prime}+\frac{1}{2} A+2 L^{\prime} .
\end{gathered}
$$

2. Wenn keine isolierten Singularitäten im Innern des Grundgebietes auftreten, liegt eine vereinfachte Situation vor, insbesondere für das Studium der wichtigen Funktionenfamilie $u \leqq u_{0}$. Im übrigen muss zunächst für isolierte Singularitäten ein entsprechendes Randverhalten untersucht werden, wobei man in erster Linie die Singularitäten mit einseitig beschränktem Verhalten betrachten wird.

3. Die Differentialgleichung (9.8): $\quad D_{0}=0$ ist bis jetzt nur für die folgenden Fälle untersucht und grundsätzlich gelöst worden:

$$
\left\{\begin{array}{l}
\text { (a) } \\
\text { (b) } \\
\text { (c) }
\end{array} \quad \begin{array}{c}
L=c_{0}+c_{1} u, \\
L=c_{0}+c_{1} e^{c_{2} u}, \quad[8],[9] .
\end{array}\right.
$$

\section{IV. Übergang zu Räumen höherer Dimension}

10. Wie bei manchen anderen Terfahren, die zunächst in der Funktionentheorie entwickelt wurden und dann in die reelle Analysis übertragen wurden, stellt sich auch hier die Frage, ob dieses Verfahren im wesentlichen auf die Ebene zu beschränken ist oder ob es sich sinnvoll auf Räume beliebiger Dimension verallgemeinern lässt. Prüft man die in II genannten Teilaufgaben, so ist es ron vornherein klar, dass es hierzu vor allem notwendig und im wesentlichen auch hinreichend ${ }^{2}$ ) ist, die erste Teilaufgabe für Räume höherer Dimension zu bewältigen, auf deren Dar-

$\left.{ }^{2}\right)$ Siehe 7, sowie [2], [10], z.B. für die Erfüllung von $D_{1} \neq 0$ durch eine Modifikation der Methode, desgl. für die Untersuchung isolierter Singularitäten bestimmten Verhaltens von $u$. 
legung wir uns im folgenden beschränken wollen. Dies wollen wir zunächst für den Fall des Raumes $\boldsymbol{C}^{n}$ der $n$ komplexen Veränderlichen (A) und sodann für den Raum $\boldsymbol{R}^{m}$ mit $m$ reellen Dimensionen (B) zeigen.

(A) 11. In einem Bereich $\mathfrak{B} \subset C^{n}$ sei eine positiv definite hermitesche Metrik $d s^{2}=a_{\alpha \bar{\beta}} d z^{\alpha} d \bar{z}^{\beta}$ gegeben. Wir übernehmen die Bezeichnungen von Schouten [11]; sei $u$ eine reellwertige zweimal stetig differenzierbare Funktion, dann führen wir mit Schouten ein: die Symbole der gewöhnlichen komplexen Ableitungen

$$
\partial_{\alpha}=\frac{\partial}{\partial z^{\alpha}}=\frac{1}{2}\left(\frac{\partial}{\partial x^{\alpha}}-i \frac{\partial}{\partial y^{\alpha}}\right), \partial_{\alpha}=\frac{\partial}{\partial \bar{z}^{\alpha}}=\frac{1}{2}\left(\frac{\partial}{\partial x^{\alpha}}+i \frac{\partial}{\partial y^{\alpha}}\right),
$$

sowie für einen Skalar $u$ die Abkürzungen:

$$
u_{\alpha}=\partial_{\alpha} u, \quad u_{\bar{\alpha}}=\partial_{\alpha}^{-} u, \quad u_{\alpha \bar{\beta}}=\partial_{\bar{\beta}} \partial_{\alpha} u,
$$

ferner die Symbole der kovarianten Ableitung $\nabla_{\alpha}, \nabla_{\bar{\alpha}}$, z. B.

$$
\begin{gathered}
\nabla_{\mu} w_{\nu}=\partial_{\mu} w_{\nu}-\Gamma_{\mu \nu}^{k} w_{k}, \Gamma_{\mu \nu}^{k}=a^{k \bar{g}} \partial_{\bar{\mu}} a_{\nu \bar{Q}}, \nabla_{\mu} w_{\bar{\nu}}=\partial_{\mu} w_{\bar{\nu}}, \\
\nabla_{\bar{\mu}} w_{\bar{\nu}}=\partial_{\bar{\mu}} w_{\bar{\nu}}-\Gamma_{\bar{\mu} \bar{\nu}}^{\bar{k}} w_{\bar{k}}, \Gamma_{\bar{\mu} \bar{\nu}}^{\bar{k}}=\bar{\Gamma}_{\mu \nu}^{k}, \nabla_{\bar{\mu}} w_{\nu}=\partial_{\bar{\mu}} w_{\nu},
\end{gathered}
$$

mit den bekannten Regeln, insbesondere:

$$
\begin{gathered}
\nabla_{\alpha} a_{\beta \bar{\gamma}}=0, \quad \nabla_{\alpha} a^{\beta \bar{\gamma}}=0, \\
\nabla_{\bar{\mu}} \nabla_{\lambda} w_{\alpha}-\nabla_{\lambda} \nabla_{\bar{\mu}} w_{\alpha}=-R_{\bar{\mu} \hat{\lambda} \alpha} \varrho w_{\varrho}
\end{gathered}
$$

mit

$$
\begin{gathered}
R_{\bar{\mu} \hat{\lambda} \alpha} \varrho=\partial_{\bar{\mu}} \Gamma_{\dot{\lambda} \alpha}^{\varrho}, \\
\nabla_{\lambda} \nabla_{\alpha} w_{\bar{\mu}}-\nabla_{\alpha} \nabla_{i} w_{\bar{\mu}}=-2 S_{\dot{\mu} \alpha} \varrho \nabla_{\varrho} w_{\bar{\mu}}
\end{gathered}
$$

mit

$$
S_{\dot{\alpha} \alpha} \underline{o}=\frac{1}{2}\left(\Gamma_{\dot{\alpha} \alpha}^{o}-\Gamma_{\alpha \hat{\alpha}}^{\varrho}\right)
$$

sowie den dazu konjugiert komplexen Formeln.

Sodann bilden wir den ersten und zweiten Beltrami-Operator

$$
\begin{gathered}
\gamma=\delta_{1} u=a^{\alpha \bar{\beta}} u_{\alpha} u_{\bar{\beta}}, \\
\delta_{2} u=a^{\alpha \bar{\beta}} u_{\alpha \bar{\beta}},
\end{gathered}
$$

und nehmen nunmehr an, dass u Lösung der folgenden partiellen Differentialgleichung

$$
\delta_{2} u=L(u, \gamma)
$$


sei. Nach dem früheren Vorbild berechnen wir nun den zweiten BeltramiOperator für die Funktion

$$
v=\gamma-f(u)
$$

wobei $f(u)$ eine zweimal stetig differenzierbare Funktion sei. Führt man diese Rechnung durch, so ergibt sich zunächst $(n>1)$ :

$$
\begin{gathered}
\delta_{2} v=a^{\alpha \bar{\beta}} v_{\alpha \bar{\beta}}=\frac{1}{\gamma} \delta_{1} v+a^{\alpha \bar{\beta}}\left(v_{\alpha} F_{\bar{\beta}}+v_{\bar{\beta}} F_{\alpha}\right) \\
+\gamma\left(-f^{\prime \prime}+2\left(L_{u}+L_{\gamma} f^{\prime}\right)\right)-L f^{\prime}+Q+H+M
\end{gathered}
$$

mit

$$
\begin{gathered}
F_{\alpha}=\frac{1}{\gamma}\left(f^{\prime} u_{\alpha}-a^{\lambda \bar{\mu}} u_{\dot{\gamma}} u_{\alpha \bar{\mu}}\right), \quad F_{\bar{\beta}}=\overline{\left(F_{\beta}\right)}, \\
Q=a^{i \bar{\mu}}{\stackrel{h}{q_{i}}{ }_{q_{\bar{\mu}}} \geqq 0}
\end{gathered}
$$

wobei die $\stackrel{h}{q}_{\lambda}, h=1, \ldots, n-1$, aus der Darstellung (11.17) $\nabla_{\lambda} u_{\alpha}=p_{\lambda} u_{\alpha}+\stackrel{h}{q} \stackrel{h}{D}_{\alpha}, \quad a^{\alpha \bar{\beta}} u_{\alpha} \stackrel{h}{D_{\bar{\beta}}}=0, \quad a^{\alpha \bar{\beta}} \stackrel{h}{D_{\alpha}} \stackrel{j}{D_{\bar{\beta}}}=\delta^{h j}$, gewonnen sind und auch über $h$ zu summieren ist, wenn $h$ zweimal auftritt, $\stackrel{h}{q_{\bar{\mu}}}=\overline{\left(\stackrel{h}{q}_{\mu}\right)}, \stackrel{h}{D_{\bar{\beta}}}=\overline{\left(D_{\beta}\right)}$.

$$
\begin{aligned}
& H=f^{\prime 2}-\frac{2}{\gamma} f^{\prime} A_{0}+\frac{1}{\gamma} A_{1}+A_{2}-A_{3}, \\
& M=-2 a^{\alpha \bar{\beta}}\left(a^{\varrho \bar{\mu}} S_{\varrho \alpha}{ }^{\lambda \lambda} u_{\bar{\beta}}+a^{\lambda \bar{\sigma}} S_{\bar{\sigma} \overline{\bar{\beta}}} \overline{\bar{u}}^{\overline{\bar{u}}} u_{\alpha}\right) u_{\overline{\bar{\nu}_{\bar{u}}}}, \\
& A_{0}=a^{\lambda \bar{\mu}} a^{\alpha \bar{\beta}} u_{\lambda} u_{\bar{\beta}} u_{\alpha \bar{\mu}} \text {, } \\
& A_{1}=a^{\lambda \bar{\mu}} a^{\alpha \bar{\beta}} a^{\tau \bar{\sigma}} u_{\alpha} u_{\bar{\sigma}} u_{\bar{\lambda} \bar{\beta}} u_{\tau \bar{\mu}} \text {, } \\
& A_{2}=a^{\lambda \bar{\mu}} a^{\alpha \bar{\beta}} u_{\alpha \bar{\mu}} u_{i \bar{\beta}} \text {, } \\
& A_{3}=a^{\alpha \bar{\beta}} a^{\lambda \bar{\mu}} R_{\bar{\mu} \dot{\lambda} \alpha}{ }^{\varrho} u_{\varrho} u_{\bar{\beta}} \text {. }
\end{aligned}
$$

Durch den Ansatz (11.17) werden die $\nabla_{\lambda} u_{\alpha}$ eliminiert, die dort vorkommenden $p_{\lambda}$ berechnen sich zu

$$
p_{\lambda}=\frac{1}{\gamma}\left(\gamma_{\lambda}-a^{\alpha \bar{\beta}} u_{\alpha} u_{\bar{\beta} \lambda}\right) \quad \text { mit } \gamma_{\lambda}=v_{\lambda}+f^{\prime} u_{\lambda},
$$

während die $\stackrel{h}{q_{\lambda}}$ nur noch innerhalb $Q$ vorkommen. 
12. Wir stellen nun an die gegebene Metrik zwei weitere Forderungen:

1.

$$
S_{\varrho \alpha}{ }^{\lambda}=0, \quad S_{\varrho} \dot{\bar{\alpha}}_{\bar{\alpha}}^{\bar{\lambda}}=0 .
$$

Dies bedeutet die Einschränkung der hermiteschen Metrik auf eine Kählersche Metrik, d. h., es gibt dann, [11], eine Skalarfunktion $\varphi$, sodass gilt:

$$
\begin{aligned}
a_{\varkappa \bar{\lambda}} & =\partial_{\bar{\lambda}} \partial_{\varkappa} \varphi . \\
R_{\alpha \bar{\beta}} & =\frac{1}{n} R a_{\alpha \bar{\beta}},
\end{aligned}
$$

wobei

$$
\begin{gathered}
R_{v \bar{\lambda}}=R_{\bar{\mu} \nu \bar{\lambda}}^{\cdots \bar{\mu}}=a^{\alpha \bar{\mu}} R_{\bar{\mu} \nu \bar{\lambda} \alpha}=\overline{R_{\bar{\nu} \lambda}}, \\
R=R_{\alpha \bar{\beta}} a^{\alpha \bar{\beta}}=\text { Skalar }
\end{gathered}
$$

der später als negative Konstante gewählt wird, dies bedeutet eine ähnliche Einschränkung der Kählerschen Metrik, wie man sie im reellen Fall der Riemannschen Metrik als Einstein-Metrik bezeichnet. Wir wollen daher eine Metrik, die die Forderungen 1 und 2 erfüllt, kurz eine Einstein-. Kähler-Metrik nennen.

Die Forderungen 1 und 2 haben zur Folge:

$$
M=0
$$

und

$$
A_{3}=-\frac{1}{n} R \gamma
$$

13. Während wir uns bei $Q$ mit der Abschätzung $Q \geqq 0$ benügen, bedarf die Abschätzung von $H$ einer ausführlichen Überlegung. Wir führen hierzu für die gemischten Ableitungen $u_{\alpha \bar{\mu}}$ die folgende Darstellung ein:

$$
u_{\alpha \bar{\mu}}=P_{\bar{\mu}} u_{\alpha}+\stackrel{h}{T_{\bar{\mu}}} \stackrel{h}{D_{\alpha}}=P_{\alpha} u_{\bar{\mu}}+\stackrel{h}{T_{\alpha}} \stackrel{h}{D_{\bar{\mu}}},
$$

wobei die $\stackrel{h}{D}_{\alpha}, \stackrel{h}{D}_{\bar{\mu}}$ bereits in (11.17) vorkommen.

Ausserdem bedienen wir uns der Abkürzungen:

$$
\left\{\begin{array}{l}
\sigma=f^{\prime}-2 L, P^{*}=a^{\lambda \bar{\mu}} P_{\lambda} P_{\bar{\mu}}, \quad \tau^{2}=a^{\lambda \mu} \stackrel{h}{T_{\lambda}} \stackrel{h}{T}_{\bar{\mu}} \\
h \\
t=a^{\alpha \bar{\beta}} a_{\alpha} T_{\bar{\beta}}^{h}, \quad p=a^{\alpha \bar{\beta}} u_{\alpha} P_{\bar{\beta}}, \quad r=a^{\alpha \bar{\beta}} T_{\bar{\beta}}^{h} D_{\alpha}, \quad s=\frac{1}{\gamma} \stackrel{h}{t}(t) \\
\operatorname{mit} \quad p=\bar{p}, \quad r=\bar{r}, L=p+r
\end{array}\right.
$$

wobei die folgenden Ungleichungen bestehen: 


$$
r^{2} \leqq(n-1) \tau^{2}
$$

$$
s \leqq \tau^{2} \text {. }
$$

Ersetzt man nun die "gemischten» 2 . Ableitungen $u_{\alpha \bar{\mu}}$ durch die oben angegebenen Ausdrücke, so erhält man

(13.5) $A_{0}=\gamma p, A_{1}=\gamma\left(p^{2}+s\right)=\gamma^{2} P^{*}, \quad$ somit $\quad \gamma P^{*}=p^{2}+s$,

$$
A_{2}=P^{*} \gamma+\tau^{2}=p^{2}+s+\tau^{2},
$$

und schliesslich:

$$
H=f^{\prime 2}-2 f^{\prime} p+2 p^{2}+2 s+\tau^{2}+\frac{R}{n} \gamma,
$$

oder wegen $p=L-r$ :

$$
H=\left(f^{\prime}-L\right)^{2}+L^{2}+\frac{R}{n} \gamma+2 s+V(r),
$$

wobei $s \geqq 0$ und

$$
V(r)=2 r^{2}+2 r \sigma+\tau^{2}
$$

unter der Nebenbedingung (13.3) nach unten abzuschätzen ist. Man erhält

$$
\left\{\begin{array}{rr}
\text { für } \quad(n-1) \tau^{2}>\frac{\sigma^{2}}{4}: & \\
\qquad(r) \geqq-\frac{1}{2} \sigma^{2}+\tau^{2} \geqq-\gamma_{1} \sigma^{2} & \text { mit } \gamma_{1}=\frac{2 n-3}{4(n-1)}, \\
\text { für } \quad(n-1) \tau^{2} \leqq \frac{\sigma^{2}}{4}: & \text { mit } \gamma_{2}=\frac{n-1}{2 n-1}>\gamma_{1},
\end{array}\right.
$$

$$
H \geqq\left(f^{\prime}-L\right)^{2}+L^{2}-\frac{n-1}{2 n-1}\left(f^{\prime}-2 L\right)^{2}+\frac{R}{n} \gamma .
$$

14. Trägt man dies nun in den ursprünglichen Ausdruck für $\delta_{2} v$ ein, so erhält man in Verbindung mit (11.16) und (12.1.c): 
$(14.1)$

$$
\delta_{2} v \geqq \frac{1}{\gamma} \delta_{1} v+a^{\alpha \bar{\beta}}\left(v_{\alpha} F_{\bar{\beta}}+v_{\bar{\beta}} F_{\alpha}\right)+D
$$

mit

(14.2) $\quad D=$

$(f+v)\left(-f^{\prime \prime}+2\left(L_{u}+f^{\prime} L_{\gamma}\right)+\frac{1}{n} R\right)+\frac{1}{2 n-1}\left(n f^{\prime}-L\right)\left(f^{\prime}-2 L\right)$.

Hierin hat man nun überall $\gamma=v+f(u) \quad$ zu setzen und $L=[L(u, \gamma)]_{\gamma=v+f}$ und ebenso $L_{u}, L_{\gamma}$ nach $v$ zu entwickeln. Man erhält auf diese Weise

$$
D=D_{0}+D_{1} v+\ldots
$$

mit

$$
f\left(-f^{\prime \prime}+\frac{R}{n}+2\left(L_{u}^{0}+f^{\prime} L_{\gamma}^{0}\right)\right)+\frac{1}{2 n-1}\left(n f^{\prime}-L^{0}\right)\left(f^{\prime}-2 L^{0}\right),
$$

$$
\begin{aligned}
D_{1}=\left(-f^{\prime \prime}+\frac{R}{n}+2\left(L_{u}^{0}\right.\right. & \left.\left.+f^{\prime} L_{\gamma}^{0}\right)\right)+2 f\left(L_{u \gamma}^{0}+f^{\prime} L_{\gamma \gamma}^{0}\right) \\
& -\frac{1}{2 n-1}\left((2 n+1) f^{\prime}-4 L^{0}\right) L_{\gamma}^{0} .
\end{aligned}
$$

Hierin bedeutet wieder wie früher der obere Index 0 , dass nachträglich das Argument $\gamma=f$ zu setzen ist.

Soll $D_{0}=0$ wieder eine Differentialgleichung für $f(u)$ sein, so ergibt sich hieraus als weitere Forderung an die Metrik: $R=$ const., sofern dies nicht bereits eine Folge von (12.2.a) ist.

Für den wichtigen Sonderfall $L_{\gamma}=0, L=L(u)$, ergibt sich insbesondere:

$$
D=D_{0}+D_{1} v
$$

mit

$$
\begin{gathered}
D_{0}=f\left(-f^{\prime \prime}+\frac{1}{n} R+2 L^{\prime}\right)+\frac{1}{2 n-1}\left(n f^{\prime}-L\right)\left(f^{\prime}-2 L\right), \\
D_{1}=-f^{\prime \prime}+\frac{1}{n} R+2 L^{\prime} .
\end{gathered}
$$

Für $n=1$ ist $R=A / 2$ zu setzen und die Ungleichungen (14.1) und (14.6) gehen in die entsprechenden Gleichungen (9.2) und (9.7) über. 
A. I. $336 / 6$

(B) 15. In einem Grundbereich $\mathfrak{B} \subset R^{m} \quad(m \geqq 2)$ sei eine positiv definite Riemannsche Metrik

$$
d s^{2}=g_{\alpha \beta} d x^{\alpha} d x^{\beta}
$$

gegeben. Wir bedienen uns des ersten und des zweiten Beltrami-Operators:

$$
\begin{aligned}
\gamma=\Delta_{1} u & =g^{\alpha \beta} u_{\alpha} u_{\beta}, \\
\Delta_{2} u & =g^{\alpha \beta} \nabla_{\beta} u_{\alpha},
\end{aligned}
$$

worin

$$
u_{\alpha}=\frac{\partial}{\partial x^{\alpha}} u
$$

ist und $\nabla_{\beta}$ den Operator der kovarianten Ableitung bedeutet. Wie früher berechnen wir nunmehr für

$$
v=\gamma-f(u)
$$

den zweiten Beltrami-Operator $\Delta_{2} v$ unter der Voraussetzung, dass $u$ der partiellen Differentialgleichung

$$
\Delta_{2} u=L(u, \gamma)
$$

genüge, und stellen an die Riemannsche Metrik die Forderung:

$$
K_{\alpha \beta}=K_{\gamma \alpha \beta} \cdots=\frac{1}{m} K g_{\alpha \beta}, \quad K=\text { const. }
$$

die Metrik sei also eine Einstein-Metrik.

Wir erhalten:

$$
=\gamma\left(-f^{\prime \prime}+\frac{2}{m} K+2\left(L_{u}+f^{\prime} L_{\gamma}\right)\right)-f^{\prime} L+2 L_{\gamma} \Delta_{1}(v, u)+q
$$

mit

$$
\Delta_{1}(v, u)=g^{\lambda \mu} v_{\lambda} u_{\mu}
$$

und

$$
q=2 g^{\alpha \beta} g^{\lambda \mu} \omega_{\alpha \lambda} \omega_{\beta \mu},
$$

worin

$$
\omega_{\alpha \lambda}=\omega_{\lambda \alpha}=\nabla_{\alpha} u_{\lambda}
$$

gesetzt ist. 
16. Wir berechnen nun $q^{*}=\min q$ unter den folgenden linearen Nebenbedingungen $(16.1)-(16.3)$ :

$$
\begin{gathered}
g^{\alpha \beta} \omega_{\alpha \beta}=L, \\
2 g^{\lambda \mu} u_{\mu} \omega_{\alpha \lambda}=\gamma_{\alpha}, \quad \alpha=1, \ldots, m, \\
\omega_{\alpha \beta}-\omega_{\beta \alpha}=0,
\end{gathered}
$$

wobei die $\omega_{\alpha \beta}$ als variabel, jedoch alle sonstigen Grössen als fest betrachtet werden. Eine etwas längere Rechnung ergibt:

$$
q \geqq q^{*}=\frac{1}{(m-1) \gamma}\left(2 L^{2} \gamma-2 L \Delta_{1}(\gamma, u)+\frac{m}{2} \Delta_{1} \gamma\right) .
$$

Im Falle $m=2$ reichen die linearen Gleichungen (16.1)-(16.3) hin, um die $\omega_{\alpha \beta} \mathrm{zu}$ eliminieren, und die Ungleichung $q \geqq q^{*}$ geht dann in eine Gleichung über.

17. Hiernach ergibt sich

$$
\Delta_{2} v \geqq \frac{m}{2(m-1)} \frac{1}{\gamma} \Delta_{1} v+\left(2 L_{\gamma}+\frac{1}{(m-1) \gamma}\left(m f^{\prime}-2 L\right)\right) \Delta_{1}(v, u)+D
$$

mit

$$
D=\gamma\left(-f^{\prime \prime}+\frac{2}{m} K+2\left(L_{u}+f^{\prime} L_{\gamma}\right)\right)+\frac{1}{m-1}\left(\frac{m}{2} f^{\prime}-L\right)\left(f^{\prime}-2 L\right) .
$$

Führt man wieder überall $\gamma=f(u)+v$ ein und entwickelt nach $v$, so erhält man schliesslich:

$$
D=D_{0}+D_{1} v+\ldots
$$

mit

$$
D_{0}=
$$

$f\left(-f^{\prime \prime}+\frac{2}{m} K+2\left(L_{u}^{0}+f^{\prime} L_{\gamma}^{0}\right)\right)+\frac{1}{m-1}\left(\frac{m}{2} f^{\prime}-L^{0}\right)\left(f^{\prime}-2 L^{0}\right)$,

$$
\begin{aligned}
D_{1}=-f^{\prime \prime}+\frac{2}{m} K+2\left(L_{u}^{0}\right. & \left.+f^{\prime} L_{\gamma}^{0}\right)+2 f\left(L_{u \gamma}^{0}+f^{\prime} L_{\gamma \gamma}^{0}\right) \\
& -\frac{1}{m-1} L_{\gamma}^{0}\left((m+1) f^{\prime}-4 L^{0}\right) .
\end{aligned}
$$

Im wichtigen Sonderfall $L_{\gamma}=0, L=L(u), L_{u}=L^{\prime}(u)$ ist:

$$
D=D_{0}+D_{1} v \text {, }
$$




$$
\begin{gathered}
D_{0}=f\left(-f^{\prime \prime}+\frac{2}{m} K+2 L^{\prime}\right)+\frac{1}{m-1}\left(\frac{m}{2} f^{\prime}-L\right)\left(f^{\prime}-2 L\right), \\
D_{1}=-f^{\prime \prime}+\frac{2}{m} K+2 L^{\prime}
\end{gathered}
$$

(im Falle $m=2$ ist $K=2 A$ ).

Damit $D_{0}=0$ wieder eine Differentialgleichung für $f(u)$ ist, hat man $\mathrm{zu}$ fordern:

$$
K=\text { const., }
$$

dies ist jedoch für $n>2$ bereits eine Folge von (15.5), [11].

Ein Vergleich mit dem Ergebnis des Falles (A) liefert übrigens für $m=2 n$ volle Übereinstimmung. Um dies einzusehen, seien etwa die Bezeichnungen des Falles (B) vorübergehend mit * versehen, ausserdem hat man die Beziehungen zu beachten:

$$
\left\{\begin{array}{l}
u^{*}=u, \Delta_{1}=4 \delta_{1}, \Delta_{2}=4 \delta_{2}, \gamma^{*}=4 \gamma, L^{*}\left(u, \gamma^{*}\right)=4 L(u, \gamma), \\
f^{*}=4 f, v^{*}=4 v, D^{*}=16 D, D_{0}^{*}=16 D_{0}, D_{1}^{*}=4 D_{1}, \\
L_{u}^{*}=4 L_{u}, L_{\gamma^{*}}^{*}=L_{\gamma}, K^{*}=4 R
\end{array}\right.
$$

18. Um weitere Betrachtungen, vor allem auch in Bezug auf das Randverhalten, zu erleichtern, wird man $K$ bzw. $R$ negativ wählen und den »vollständigen» Grundbereich der Metrik zugrunde legen, so dass sein Rand im Sinne der Metrik von einem inneren Punkt unendlich weit entfernt ist. Der Fall $u \leqq u_{0}$ ohne das Auftreten von im Innern des Grundbereiches der Metrik gelegenen Singularitäten von $u$ dürfte wohl der einfachste Fall sein, der sich zunächst der Behandlung anbietet, während in anderen Fällen ein Studium der isolierten Singularitäten von $u$ vorausgehen muss.

Für die Integration der Differentialgleichung $D_{0}=0$ erhält man aus dem klassischen Fall der Ebene verschiedene wertvolle Hinweise, die die Integration erleichtern. Zum Beispiel findet man im Falle (A), $R<0$ $(f>0)$, für

$$
\begin{gathered}
\xi(u)=\left(f^{\prime}-2 L(u, f)\right) f^{-1 / 2}, \\
\xi^{\prime}=f^{-1 / 2}\left(\frac{R}{n}+\frac{1}{2(2 n-1)} \xi^{2}\right),
\end{gathered}
$$

hieraus ergeben sich die beiden partikulären Zwischenintegrale:

$$
\xi= \pm c \text { mit } c=\sqrt{\frac{-2 R(2 n-1)}{n}} .
$$


Für jedes sonstige Integral, das nicht in diesen Zwischenintegralen enthalten ist, kann man mit grossem Vorteil für $f(u)$ die neue Parameterdarstellung einführen:

$$
\sqrt{f}=g=g(t), \quad u=u(t),
$$

unter Verwendung des Parameters

$$
t=\frac{1}{c} \xi .
$$

Dann schreibt sich die Differentialgleichung (14.4): $D_{0}=0$ in folgendes System um:

$$
\left(t^{2}-1\right) \frac{d u}{d t}=a g
$$

$$
\left(t^{2}-1\right) \frac{d g}{d t}=(2 n-1) t g+a L^{0}, \quad a=\sqrt{\frac{2 n(2 n-1)}{-R}}, L^{0}=L\left(u, g^{2}\right)
$$

Insbesondere kann der Fall

$$
L(u, \gamma)=c_{0}+c_{1} u+c_{2} \sqrt{\gamma},
$$

an Hand des in diesem Fall linearen Systems (18.6) - (18.7) studiert werden.

19. Die dargelegte Methode ist übrigens auch im funktionentheoretischen Bereich einer weiteren Verallgemeinerung auf höhere Differentialinvarianten fähig.

Zusammenfassend lässt sich sagen, dass ihr Grundgedanke jeweils für solche Funktionenfamilien neue Abschätzungen liefert, die durch ein gewisses "Randverhalten» (im allgemeinen Sinn der obigen Darlegungen) gekennzeichnet werden können, wobei diese Methode es gestattet, aus dem Randverhalten gewisse Ungleichungen, die im Innern gelten, zu gewinnen bzw. bereits vorhandene Ungleichungen, wie z. B. $u \leqq u_{0}$, zu verschärfen. Unter diesem Gesichtspunkt kann man wohl sagen, dass sie eine Verallgemeinerung des Schwarzschen Lemmas darstellt und eine gewisse Verwandtschaft mit der Grundidee des Phragmén-Lindelöfschen Prinzips aufweist, [3].

Universität Bonn

Deutschland 


\section{Literatur}

[1] Ahlfors, L. V.: An extension of Schwarz's lemma. - Trans. Amer. Math. Soc. 43 , 1938, s. $359-364$.

[2] Bauer, K. W.: Über die Abschätzungen von Lösungen gewisser partieller Differentialgleichungen vom elliptischen Typus. - Bonn. Math. Schr. 10, 1960.

[3] Bauer, K. W., und E. Peschl: Über ein Verfahren zur Abschätzung von Lösungen der elliptischen partiellen Differentialgleichung $\delta_{2} u=L\left(u, \delta_{1} u\right)$. - Erscheint demnächst in Bayer. Akad. Wiss. Math.-Nat. Kl. S.-B.

[4] Peschl, E.: Über die Krümmung von Niveaukurven bei der konformen Abbildung einfachzusammenhängender Gebiete auf das Innere des Einheitskreises. - Math. Ann. 106, 1932, S. 574-594.

[5] -»- Über eine gewisse Funktionalgleichung und ihre Bedeutung im Blochschen Problemkreis. - Internat. Math. Nachr. 21/22, 1952, S. 31-32.

[6] -»- Les invariants différentiels non holomorphes et leur rôle dans la théorie des fonctions. - Rend. Sem. Mat. Messina 1, 1955, S. 100-108.

[7] -»- Unverzweigte konforme Abbildungen des Einheitskreises. - Erscheint demnächst in der Math. Z.

[8] Peschl, E., und K. W. Bauer: Über eine nichtlineare Differentialgleichung 2. Ordnung, die bei einem gewissen Abschätzungsverfahren eine besondere Rolle spielt. - Forschungsberichte des Landes Nordrhein-Westfalen 1306, Westdeutscher Verlag, Köln/Opladen, 1963.

[9] -»- -»- Über nichtlineare Differentialgleichungen 2. Ordnung, die für eine Abschätzungsmethode bei partiellen Differentialgleichungen vom elliptischen Typus besonders wichtig sind. - Forschungsberichte des Landes Nordrhein-Westfalen 1374, Westdeutscher Verlag, Köln/Opladen, 1963.

[10] RaUPACH, E.: Eine Abschätzungsmethode für die reellwertigen Lösungen der Differentialgleichung $\Delta \alpha=-\frac{4}{(1-z \bar{z})^{2}}$. Bonn. Math. Schr. 9; 1960.

[11] Schouten, J. A.: Ricci-calculus. An introduction to tensor analysis and its geometrical applications. - 2. Aufl., Grundlehren der mathematischen Wissenschaften X, Springer-Verlag, Berlin/Göttingen/Heidelberg, 1954. 